\title{
Fading of modern Prussian blue pigments in linseed oil medium $\dagger$
}

\author{
Louise Samain, ${ }^{* a}$ Geert Silversmit, ${ }^{b}$ Jana Sanyova, ${ }^{c}$ Bart Vekemans, ${ }^{b}$ Hélène Salomon, ${ }^{a}$ Bernard Gilbert, ${ }^{d}$ \\ Fernande Grandjean, ${ }^{e}$ Gary J. Long, ${ }^{f}$ Raphaël P. Hermann, ${ }^{g e}$ Laszlo Vincze $^{b}$ and David Strivay ${ }^{a}$
}

\author{
Received 12th December 2010, Accepted 29th March 2011 \\ DOI: $10.1039 / \mathrm{c0ja00234h}$
}

The fading of modern laboratory-synthesized and commercial Prussian blue, iron(III) hexacyanoferrate (II), based pigments in a linseed oil medium during exposure to light has been investigated. The Prussian blue pigments were painted with linseed oil, as a pure pigment and mixed with white lead, $\left(\mathrm{PbCO}_{3}\right)_{2} \mathrm{~Pb}$ $(\mathrm{OH})_{2}$, zinc white, $\mathrm{ZnO}$, or titanium white, $\mathrm{TiO}_{2}$, pigment. The samples were subjected to accelerated ageing for $800 \mathrm{~h}$ and the light fastness of the Prussian blue pigment was evaluated by reference to blue wool standards. Pure Prussian blue is extremely light fast whilst it strongly fades when mixed with a white pigment, especially with lead white or zinc oxide. The painted samples were studied by UV-visible, iron K-edge X-ray absorption, iron-57 transmission Mössbauer, and attenuated total reflectance infrared spectroscopy. X-ray absorption results reveal a decrease in the iron coordination number in aged samples in the presence of white pigment. The Mössbauer spectra of the pure Prussian blue and the unaged and aged mixtures of Prussian blue and lead white or zinc oxide at $1: 100$ and $1: 10$ dilution ratios, respectively, indicate the presence of iron(II) and iron(III) in a ratio close to one as expected for the bulk stoichiometric $\mathrm{KFe}^{\mathrm{II}}\left[\mathrm{Fe}^{\mathrm{II}}(\mathrm{CN})_{6}\right]$; no change in the spectral parameters was observed upon ageing. Combined with the X-ray near edge absorption and infrared studies, these results suggest reduction of the surface iron ions in the Prussian blue with ageing upon exposure to light.

\section{Introduction}

Prussian blue was accidentally discovered in Berlin in 1704 and eventually shown to contain a hydrated iron(III) hexacyanoferrate(II) anion, $\left\{\mathrm{Fe}^{\mathrm{III}}\left[\mathrm{Fe}^{\mathrm{II}}(\mathrm{CN})_{6}\right] \cdot x \mathrm{H}_{2} \mathrm{O}\right\}^{-}$, with varying values of $x$ up to 16 , and various alkali cations, such as $\mathrm{K}^{+}, \mathrm{NH}_{4}^{+}$ or $\mathrm{Na}^{+}$. An alkali-free Prussian blue $\mathrm{Fe}_{4}^{\mathrm{III}}\left[\mathrm{Fe}^{\mathrm{II}}(\mathrm{CN})_{6}\right]_{3} \cdot x \mathrm{H}_{2} \mathrm{O}$, which must have an increased amount of iron(III) in order to maintain charge balance, is commonly referred to as an "insoluble" Prussian blue, whereas those with alkali cations are referred to as "soluble" Prussian blues. Although all Prussian

${ }^{a}$ Centre Européen d'Archéométrie, University of Liège B15, B-4000 Sart-Tilman, Belgium.E-mail: louise.samain@ulg.ac.be; Fax: +324366 2884; Tel: +3243663696

${ }^{b} X$-ray Microspectroscopy and Imaging, Department of Chemistry, University of Ghent, B-9000 Gent, Belgium

'Institut Royal du Patrimoine Artistique, B-1000 Brussels, Belgium

${ }^{d}$ Department of Chemistry, University of Liège, B6, B-4000 Sart-Tilman, Belgium

${ }^{e}$ Department of Physics, University of Liège, B5, B-4000 Sart-Tilman Belgium

${ }^{f}$ Department of Chemistry, Missouri University of Science and Technology, University of Missouri, Rolla, MO, 65409-0010, USA

${ }^{g} J$ Julich Centre for Neutron Science JCNS and Peter Grünberg Institut PGI, JARA-FIT, Forschungszentrum Jülich GmbH, D-52425 Jülich, Germany $\dagger$ This article is part of a themed issue highlighting the latest research in the area of synchrotron radiation in art and archaeometry. blues are highly insoluble, the soluble appellation refers to the ease with which a "soluble" Prussian blue is dispersed in an aqueous solution to form a colloidal suspension. Because of both its low cost and highly intense colour, Prussian blue enjoyed immediate popularity among artists and was widely used as a pigment in paintings until 1970 . However, the permanence of Prussian blue had already been questioned by the mid-eighteenth century, because it shows a tendency to fade upon exposure to light, especially when mixed with white pigments. ${ }^{1,2}$

Although Prussian blue, and other transition metal hexacyanometallates, known as Prussian blue analogues, have been extensively studied because of their interesting magnetic, ${ }^{3,4}$ electrochemical $^{5,6,7}$ and biochemical ${ }^{8,9}$ properties, little attention has been devoted to the degradation of Prussian blues in paint layers containing binders and extenders. A better understanding of the mechanisms associated with this degradation will help in the preventive conservation and restoration of paintings that have used Prussian blues.

The intense colour of Prussian blue arises from an intervalent charge transfer between the iron(II) and iron(III) oxidation states when light is absorbed at $680 \mathrm{~nm} .{ }^{10}$ Thus, any colour fading or change corresponds to a disruption in the electron transfer pathway in a Prussian blue, i.e., a $\mathrm{Fe}^{\mathrm{III}}-\mathrm{N}-\mathrm{C}-\mathrm{Fe}^{\mathrm{II}}$ bonding pathway.

The electrochromic character ${ }^{11}$ of Prussian blue pigments has been proposed as an explanation for their fading in paint layer. 
Indeed, Prussian blue can be either electrochemically oxidized to form an iron(III) ferricyanide, known as a Prussian yellow, or reduced to iron(II)ferrocyanide, known as a Berlin white or Everitt's salt. ${ }^{12}$ These redox reactions have been extensively studied in electrochemically synthesized Prussian blue films both in the presence of an electrolyte ${ }^{5}$ and in the solid state. ${ }^{13,14}$ Photooxidation and photoreduction have also been reported in nanoparticles containing Prussian blue and a photoresponsive material, e.g., the photoconductive $\mathrm{CdS}$ semiconductor or photochromic azobenzene molecules. ${ }^{15,16}$ Such compounds can supply electrons upon UV/visible irradiation and induce redox processes in Prussian blue. However, such reactions in Prussian blue films or nanoparticles are reversible, whereas in paint layers a reversible fading is only observed in the short term; after a long exposure to light an irreversible colour change occurs in paintings. ${ }^{1}$

Any ion exchange occurring in Prussian blue could also hinder the intervalent charge transfer and lead to a loss of colour. A substitution reaction of the weakly bonded nitrogen-coordinated iron(III) ions by other metal ions has been observed during the electrochemical oxidation of Prussian blue in aqueous solutions. ${ }^{17}$

In order to identify the changes in the local electronic and structural configurations of the iron(II) and iron(III) ions in aged Prussian blue pigments, the pigments in a linseed oil matrix, have been studied by attenuated total reflection Fourier transform infrared, optical, iron K-edge X-ray absorption, and iron-57 transmission Mössbauer spectroscopy. It should be noted that all these techniques, except transmission Mössbauer spectroscopy and, to a lesser extent, X-ray absorption at $7.1 \mathrm{keV}$, are surface sensitive and provide little or no information about the bulk of the pigments. In contrast, because of the high energy of the 14.4 $\mathrm{keV} \gamma$-ray used in iron-57 transmission Mössbauer spectroscopy, it is a bulk technique that has little if any surface sensitivity. Finally, it should be noted that the last two techniques are ironelement specific techniques.

\section{Experimental}

\section{Sample preparation}

All reagents used herein for the synthesis of Prussian blue were of reagent grade quality and obtained from Sigma-Aldrich, Steinheim, Germany. Soluble Prussian blue $\mathrm{KFe}^{\mathrm{III}}\left[\mathrm{Fe}^{\mathrm{II}}(\mathrm{CN})_{6}\right]$ was synthesized according to the so-called indirect method. Aqueous solutions of $0.1 \mathrm{M} \mathrm{FeCl}_{2} \cdot 4 \mathrm{H}_{2} \mathrm{O}$ and $0.1 \mathrm{M} \mathrm{K}_{4} \mathrm{Fe}(\mathrm{CN})_{6} \cdot 3 \mathrm{H}_{2} \mathrm{O}$ were mixed at $50{ }^{\circ} \mathrm{C}$. The $\mathrm{pH}$ of the solution was adjusted to 2 by adding hydrochloric acid. A Berlin white, iron(II) ferrocyanide, precipitate was formed and aged for two hours at $90{ }^{\circ} \mathrm{C}$. The Berlin white was then oxidized with $5 \mathrm{ml} \mathrm{H}_{2} \mathrm{O}_{2}$ into $\mathrm{KFe}^{\mathrm{III}}\left[\mathrm{Fe}^{\mathrm{II}}(\mathrm{CN})_{6}\right] \cdot x \mathrm{H}_{2} \mathrm{O}$, Prussian blue. The precipitate was then thoroughly washed with deionized water and collected by centrifugation. The pigment was oven dried at $55^{\circ} \mathrm{C}$ for one hour and then ground into a fine powder. Commercial soluble and insoluble Prussian blues were also purchased both from SigmaAldrich and from Winsor \& Newton, London, England, a supplier of artists materials. The particle size of the laboratorysynthesized and commercial Prussian blues was determined with a JEOL 7500F field-emission electronic scanning microscope at the University of Namur, Belgium. All Prussian blues were of extremely fine particle size, approximately 50 to $200 \mathrm{~nm}$ in diameter.

Both because the value of $x$ in the soluble and insoluble Prussian blues, $\mathrm{KFe}^{\mathrm{III}}\left[\mathrm{Fe}^{\mathrm{II}}(\mathrm{CN})_{6}\right] \cdot x \mathrm{H}_{2} \mathrm{O}$ and $\mathrm{Fe}_{4}{ }^{\mathrm{III}}$ $\left[\mathrm{Fe}^{\mathrm{II}}(\mathrm{CN})_{6}\right]_{3} \cdot x \mathrm{H}_{2} \mathrm{O}$, has not been determined, and because the amount of water present is expected to play little role in any fading of colour, the $x \mathrm{H}_{2} \mathrm{O}$ in the formulation is not included in the discussion below.

These above powders were mixed with boiled linseed oil and painted onto a $2 \mathrm{~cm}^{2}$ universally primed $100 \%$ linen canvas. Because pure Prussian blue has such a deep colour it was rarely used by artists in the pure form. Thus the samples were also diluted with white pigments obtained from Kremer Pigmente $\mathrm{GmbH} \& \mathrm{Co} \mathrm{KG}$, Alchstetten, Germany or provided by the Royal Institute for Cultural Heritage in Brussels, Belgium. The specific white pigments used were zinc oxide, $\mathrm{ZnO}$, titanium dioxide, $\mathrm{TiO}_{2}$, and lead white. Lead white, which was the most popular white pigment used in the eighteenth and nineteenth centuries is basic lead carbonate, which can have a somewhat variable composition but is typically close to $\left(\mathrm{PbCO}_{3}\right)_{2} \mathrm{~Pb}(\mathrm{OH})_{2}$; in nature it is found as the mineral hydrocerussite. Lead white particles are of uniform size in the range of 1 to $2 \mu \mathrm{m}$ in diameter. Zinc oxide, first used as a pigment by artists in the late eighteenth century, is still in use. It is characterized by particles of 0.25 to $1 \mu \mathrm{m}$ in diameter. The rutile form of titanium dioxide has been the most popular white pigment since 1945 . Currently produced pigmentary rutile is made up of pigment particles from 0.2 to 0.5 $\mu \mathrm{m}$ in diameter. Samples containing, weight to weight, Prussian blue pigment to white $1: 1,1: 10$, and $1: 100$ dilution ratios were prepared. The amount of linseed oil needed for each mixture was determined by considering the oil absorption indexes of Prussian blue, lead white, zinc white, and titanium white, which are 100,8 to 12,13 to 22 , and 15 to $20 \mathrm{wt} \%$, respectively. ${ }^{18}$ The density of Prussian blue painted with lead white in $1: 1,1: 10$ and $1: 100$ dilution ratios are approximately 1.6, 0.7 and $0.04 \mathrm{mg} \mathrm{cm}^{-2}$, respectively. Paint layers prepared with zinc oxide or titanium dioxide in $1: 10$ dilution ratio contain a density of Prussian blue of approximately $0.7 \mathrm{mg} \mathrm{cm}^{-2}$. In order to evaluate the light fastness of the white pigments used, paint layers containing only white pigments have also been prepared. The thickness of the paint layers was determined with an Olympus BX51 optical microscope from cross-sections of millimetre size fragments of the paint layers that were embedded in resin and carefully polished; the painted samples are all approximately $20 \mu \mathrm{m}$ thick.

\section{Accelerated ageing}

The samples were subjected to accelerated ageing up to $800 \mathrm{~h}$, or almost five weeks, by using a SUNTEST CPS+ weathering chamber located at the Royal Institute for Cultural Heritage at Brussels, Belgium. The chamber is equipped with a xenon lamp and a window glass filter that removed UV radiation below 320 $\mathrm{nm}$ was used to simulate outdoor ageing, as recommended in ISO 105-B04. A ventilation system was used to cool the ageing chamber to a constant temperature of about $35{ }^{\circ} \mathrm{C}$. The luminance at the surface of the sample was approximately 120000 lux. During the ageing process half of the painted canvas surface 
area was covered with aluminium to serve as reference after ageing. In order to quantify the permanence of the pigments, the samples were aged together with blue wool light fastness reference standards as specified in ISO $104 \mathrm{~B}$, a standard that is used by the pigment industry for determining the resistance of colour to the action of artificial light. During the first $400 \mathrm{~h}$ of weathering the samples were removed from the weathering chamber every $50 \mathrm{~h}$ for about $1 \mathrm{~h}$ in order to perform colorimetric measurements which could not be carried out in the chamber. After $400 \mathrm{~h}$ of light exposure, the samples were removed from the chamber and kept in the dark for one week in order to determine whether there was a return of the original colour of the samples after dark storage, a phenomenon that has been reported earlier. ${ }^{19}$ After one week in the dark, the ageing was continued under the same conditions as described above with colorimetric measurements every 100 to $150 \mathrm{~h}$.

\section{Colour measurement}

The colour of each sample was measured before the start of ageing and then periodically during light exposure by means of a BYK-Gardner colour guide. This instrument has a $45^{\circ} / 0^{\circ}$ geometry and a $4 \mathrm{~mm}$ aperture. A visible spectrum was recorded between 400 and $700 \mathrm{~nm}$ with a resolution of $20 \mathrm{~nm}$, and automatically converted to the Commission Internationale de l'Eclairage 1976 unitless $L^{*}, a^{*}$, and $b^{*}$ parameters by using the standard illuminant D65 as a reference. These parameters correspond to the lightness, red-greeness, and yellow-blueness, respectively, of the colour and are derived from the XYZ tristimulus values of a reference white object and the coloured object. ${ }^{20}$ The colour difference, $\Delta E^{*}$, between the unexposed and the aged exposed portion of the sample was calculated from:

$$
\Delta E^{*}=\left[\left(\Delta L^{*}\right)^{2}+\left(\Delta a^{*}\right)^{2}+\left(\Delta b^{*}\right)^{2}\right]^{1 / 2}
$$

Typically, a $\Delta E^{*}$ colour difference of less than one is imperceptible to the human eye. The evolution of $\Delta E^{*}$ rather than $\Delta b^{*}$ is used to assess the fading of Prussian blue in order to take into account the three parameters $L^{*}, a^{*}$ and $b^{*}$. Indeed a change is expected in the yellow-blueness $\Delta b^{*}$ but also in the lightness $\Delta L^{*}$.

Samples were also analyzed by UV/visible reflectance spectroscopy with a StellarNet EPP2000C spectrometer equipped with a CCD detector. The optical-fiber probe consists of six illuminating fibers and a single fiber that collects the reflected light. The sample is illuminated over a surface area of approximately $4 \mathrm{~mm}^{2}$ at an angle of $45^{\circ}$ in order to avoid direct reflection; a Halon D50 white reference was used for calibration. The spectra were recorded in reflection mode between 350 and 880 $\mathrm{nm}$, with a resolution of $1 \mathrm{~nm}$. The absorbance spectra were then calculated with the SpectraWiz software.

\section{X-ray absorption spectroscopy}

The iron K-edge X-ray near edge absorption spectroscopy, XANES, and extended X-ray fine structure spectroscopy, EXAFS, experiments were performed at the DUBBLE DutchBelgian beamline at the European Synchrotron Radiation Facility in Grenoble, France. This instrument is located at a bending magnet port of the electron storage ring, with a magnetic field strength of $0.4 \mathrm{~T}$ and a critical energy of $9.6 \mathrm{keV}$, and is equipped with a $\mathrm{Si}(111)$ double crystal monochromator that has a relative energy resolution, $\Delta E / E$, of $2 \times 10^{-4}$. $^{21}$ The higher harmonics were suppressed with a Si reflecting strip on a mirror after the monochromator. The energy scale was calibrated with a $4 \mu \mathrm{m}$ thick iron foil recorded in transmission mode; the energy of the first maximum in the derivative of the iron $\mathrm{K}$-edge absorption was taken at $7112 \mathrm{eV}$. The intensities of the incident and transmitted X-ray beams were measured with Oxford Instrument ionisation detectors. Because of the low concentration of iron in the diluted samples, the iron K-edge spectra were recorded in fluorescence mode. The fluorescence spectra were collected with a liquid nitrogen cooled energy dispersive nine channel monolithic Ge detector. Powders of both the laboratory-synthesized and commercial Prussian blue samples have been measured in transmission mode. After appropriate mixing and grinding with boron nitride, the powders were pressed into self-supporting pellets in a stainless steel sample holder.

The EXAFS data reduction and analysis were performed with the XDAP software. ${ }^{22}$ The pre-edge background in the X-ray absorption spectra was subtracted by a modified Victoreen curve $^{23}$ for transmission data and by a linear function or a constant for the spectra recorded in fluorescence detection mode. The atomic background, $\mu_{0}$, was subtracted with a cubic spline routine. ${ }^{24}$ The pre-edge background subtracted spectra were normalised to the edge jump, which was taken to be the value of the atomic background at $50 \mathrm{eV}$ above the K-edge. The fit parameters were determined by multiple shell fitting in $\mathrm{R}$-space, by applying the difference file technique using Fourier transformations. ${ }^{25}$ The phase shifts and backscattering amplitudes used in the EXAFS analysis were obtained from FEFF8.0 calculations ${ }^{26}$ on the Prussian blue crystal structure, by taking the $\mathrm{Fe}^{\mathrm{II}}$ ion as the central iron site. The FEFF output for the shortest single scattering path, $\mathrm{Fe}^{\mathrm{II}}-\mathrm{C}$, was used to model the first coordination shell around the $\mathrm{Fe}$ sites in the Prussian blue structure, i.e., $\mathrm{Fe}{ }^{\mathrm{II}}-\mathrm{C}$ or $\mathrm{Fe}^{\mathrm{III}}-\mathrm{N}$. Identical scattering and amplitude functions were used for $\mathrm{N}$ and $\mathrm{C}$, as the scattering properties for neighbouring atoms in the periodic table are similar. Due to the linear geometry of the $\mathrm{Fe}^{\mathrm{II}}-\mathrm{C}-\mathrm{N}-\mathrm{Fe}^{\mathrm{III}}$ path in the Prussian blue structure, the contributions of the multiple scattering for the second and third shells are stronger than the single scattering contributions for these shells. ${ }^{27}$ Therefore, the multiple scattering paths with the highest probability were retained to model the second and third shells. For the second shell, $\mathrm{Fe}^{\mathrm{II}}-\mathrm{N}$ or $\mathrm{Fe}^{\mathrm{III}}-\mathrm{C}$, this was a 3-leg path, $\mathrm{Fe}^{\mathrm{II}}-\mathrm{C}-\mathrm{N}-\mathrm{Fe}^{\mathrm{II}}$ and for the third shell, $\mathrm{Fe}^{\mathrm{II}}-\mathrm{Fe}^{\mathrm{III}}$, this was a 4-leg path, $\mathrm{Fe}^{\mathrm{II}}-\mathrm{Fe}^{\mathrm{III}}-\mathrm{N}-\mathrm{C}-\mathrm{Fe}^{\mathrm{II}}$. The $\mathrm{X}$-ray absorption spectroscopy probes an average over the two $\mathrm{Fe}^{\mathrm{II}}$ and $\mathrm{Fe}^{\mathrm{III}}$ sites, within the Prussian blue. This average coordination for commercial and laboratorysynthesized soluble Prussian blue is given in Table $1 .{ }^{28}$

The accuracy of the phase and backscattering functions for the first and second shells obtained in this way was evaluated with a fit on the first two coordination shells in a $\mathrm{K}_{4}\left[\mathrm{Fe}(\mathrm{CN})_{6}\right]$ powder sample measured in transmission. The FEFF8.0 calculations were loaded into the XDAP software and $\mathrm{S}_{0}^{2}$ and Debye-Waller factors were optimised to obtain agreement with the crystal coordination numbers. The Debye-Waller factors reported in this work for the Prussian blue samples are, therefore, relative to the $\mathrm{K}_{4}\left[\mathrm{Fe}(\mathrm{CN})_{6}\right]$ structure. The bond distances were optimised in 
Table 1 Average bond distances, $R$, coordination numbers, $N$, Debye-Waller factors $\Delta \sigma^{2}$, and inner potential corrections, $\Delta E_{0}$, for the EXAFS fits on the commercial and laboratory-synthesized soluble Prussian blue powder samples

\begin{tabular}{|c|c|c|c|c|c|c|}
\hline Absorber-scatter & \multicolumn{3}{|c|}{ Crystal structure } & \multicolumn{3}{|c|}{ EXAFS fit values } \\
\hline $\mathrm{Fe}(\mathrm{III})-\mathrm{N}$ & $2.04(1)$ & 3 & $2.06(1)$ & $3.1(1)$ & $0.0002(2)$ & $2.2(5)$ \\
\hline $\mathrm{Fe}(\mathrm{II})-\mathrm{N}$ & $3.04(1)$ & 3 & $3.04(1)$ & $2.9(1)$ & $0.0002(2)$ & $1.7(5)$ \\
\hline $\mathrm{Fe}(\mathrm{III})-\mathrm{C}$ & $3.16(1)$ & 3 & $3.15(1)$ & $3.0(2)$ & $0.0002(2)$ & $4.3(6)$ \\
\hline $\mathrm{Fe}(\mathrm{II})-\mathrm{Fe}(\mathrm{III})$ & $5.08(1)$ & 6 & $5.10(1)$ & $6.1(2)$ & $-0.0005(2)$ & $-4.6(3)$ \\
\hline
\end{tabular}

the fit and deviated by at most $0.01 \AA$ from the average bond distances reported in the $\mathrm{K}_{4}\left[\mathrm{Fe}(\mathrm{CN})_{6}\right]$ crystal structure. ${ }^{29} \mathrm{~A}$ similar procedure was followed for the third shell, by fitting the Fe-Fe shell with a coordination number, $N$, of 6 for the averaged spectrum of soluble Prussian blue powder samples.

In insoluble Prussian blue, every fourth $\mathrm{Fe}^{\mathrm{II}}$ site is vacant compared to the soluble crystal structure, resulting in a lower theoretical Fe-Fe coordination number of $N=5.14$. However, no significant decrease in coordination number was observed for the insoluble reference samples available. The EXAFS spectra for both soluble and insoluble Prussian blue powder samples could be fitted with the above described phase and backscattering functions and resulted in values close to the crystal X-ray structure values, see Table 1 . One common Debye-Waller factor was considered for the four contributions within the first two coordination shells. For all EXAFS fits, the coordination numbers for the two contributions in the second $\mathrm{Fe}^{\mathrm{II}}-\mathrm{N}$ and $\mathrm{Fe}^{\mathrm{III}}-\mathrm{C}$ shells were constrained to the same value. As these contributions are fitted with the 3-leg multiple scattering paths involving both the $\mathrm{C}$ and $\mathrm{N}$ atoms in the first and second shell, the two different 3-leg paths will both disappear as soon as a $\mathrm{C}$ or $\mathrm{N}$ atom in the first or second coordination shell disappears. So the same coordination number for the two 3-leg paths can be assumed. The coordination numbers for the contributions in the first coordination shell however were not constrained.

\section{Mössbauer spectroscopy}

The iron-57 Mössbauer spectra have been measured between 85 and $295 \mathrm{~K}$ in a Janis Supervaritemp cryostat with a constantacceleration spectrometer which utilized a rhodium matrix cobalt-57 source and was calibrated at $295 \mathrm{~K}$ with $\alpha$-iron powder. The Mössbauer spectral absorbers of the pure laboratory-synthesized Prussian blue contained $10 \mathrm{mg} \mathrm{cm}^{-2}$ of powdered sample mixed with boron nitride. The Mössbauer spectral absorbers of pristine unaged $\mathrm{KFe}^{\mathrm{III}}\left[\mathrm{Fe}^{\mathrm{II}}(\mathrm{CN})_{6}\right]$, and unaged and aged mixtures with white lead, $\left(\mathrm{PbCO}_{3}\right)_{2} \mathrm{~Pb}(\mathrm{OH})_{2}$, and zinc white, $\mathrm{ZnO}$, consisted of a $20 \mu \mathrm{m}$ layer on canvas. Depending on the dilution, the thickness of Prussian blue in the paint layer varies from 0.07 to $0.7 \mathrm{mg} \mathrm{cm}^{-2}$. All spectra presented herein have been fitted with two Lorentzian single lines, i.e., a total of six spectral parameters were adjusted, two isomer shifts, $\delta^{\mathrm{II}}$ and $\delta^{\mathrm{II}}$, two full line widths at half-height, $\Gamma^{\mathrm{II}}$ and $\Gamma^{\mathrm{III}}$, one percent area, $A^{\mathrm{II}}$, with $A^{\mathrm{III}}=100-A^{\mathrm{II}}$, and one total absorption area. Attempts to fit the spectra with two quadrupole doublets or one singlet and one doublet ${ }^{30}$ lead to quadrupole splittings that were virtually zero and/or to line widths that were smaller than the minimum natural line width of $0.195 \mathrm{~mm} \mathrm{~s}^{-1}$ and, hence, were not meaningful.

\section{Fourier transform infrared}

Painted unaged and aged samples were studied by attenuated total reflectance Fourier transform infrared spectroscopy by using a Bruker Hyperion 3000 FT-IR microscope, located at the Royal Institute for Cultural Heritage in Brussels, Belgium. The attenuated total reflectance objective is equipped with a germanium crystal. By knowing the wavelength, the refractive indices of germanium and Prussian blue, and the incidence angle, the depth of penetration was found ${ }^{13}$ to be approximately $1 \mu \mathrm{m}$. The contact surface between the germanium crystal and the sample is approximately $100 \mu \mathrm{m}^{2}$. All spectra result from the sum of 64 scans at a resolution of $4 \mathrm{~cm}^{-1}$ between 4000 and $600 \mathrm{~cm}^{-1}$. The spectra were baseline-corrected and no further ATR corrections were applied. The samples were analyzed at several spots, in order to both ensure reproducibility and to evaluate the homogeneity of the paint layer.

\section{Results}

\section{Light fastness of Prussian blue}

The light fastness of the soluble $\mathrm{KFe}^{\mathrm{III}}\left[\mathrm{Fe}^{\mathrm{II}}(\mathrm{CN})_{6}\right]$ and insoluble $\mathrm{KFe}^{\mathrm{III}}\left[\mathrm{Fe}^{\mathrm{II}}(\mathrm{CN})_{6}\right]_{3}$ Prussian blue samples range from 0 , for very poor or fugitive, to 8 for extremely good light fastness as determined by a comparison with the light fastness of the blue wool standards. The evolution of the colour change with light exposure for the laboratory-synthesized soluble Prussian blue, the commercial soluble Prussian blue, the commercial insoluble Prussian blue and the blue wool standard, is shown in Fig. 1. After $800 \mathrm{~h}$, or almost 5 weeks, of light exposure no discoloration or fading was observed for pure soluble Prussian blue samples and, thus, one can conclude that pure Prussian blue is as light fast as the blue wool standards 7 and 8. In contrast, soluble Prussian blue mixed with white pigments exhibited extensive fading. In the mixture with lead white, $\left(\mathrm{PbCO}_{3}\right)_{2} \mathrm{~Pb}(\mathrm{OH})_{2}$, it is noteworthy that discoloration increased with the dilution. In this case, the paint layer containing Prussian blue and lead white in a $1: 100$ ratio was so extensively faded that the ageing experiments were discontinued after $400 \mathrm{~h}$. The rating of light fastness of Prussian blue mixed with lead white progressively decreases as the proportion of white pigment increases: The paint layer prepared 


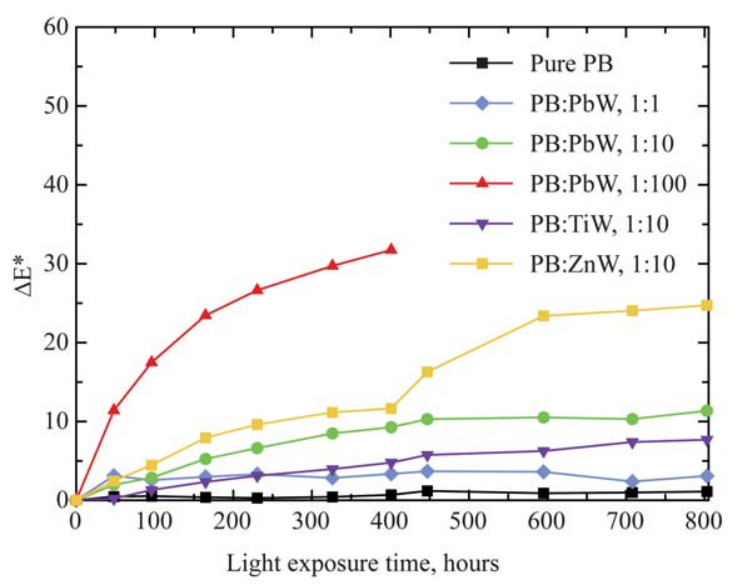

(a)

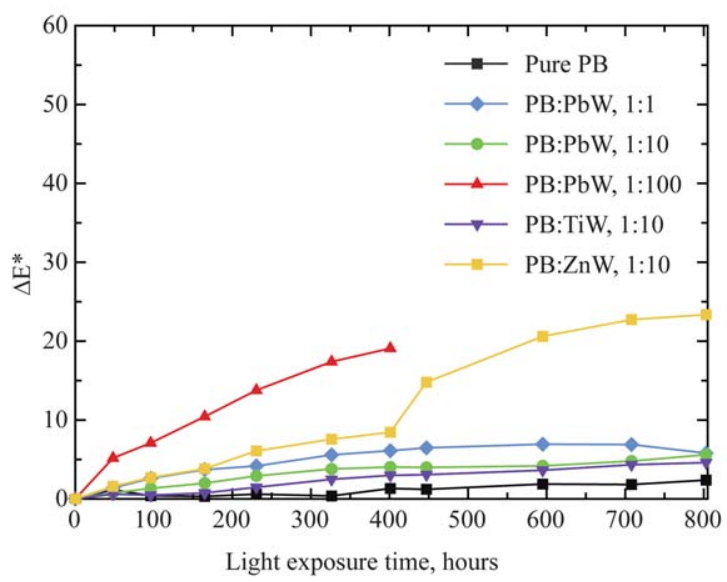

(c)

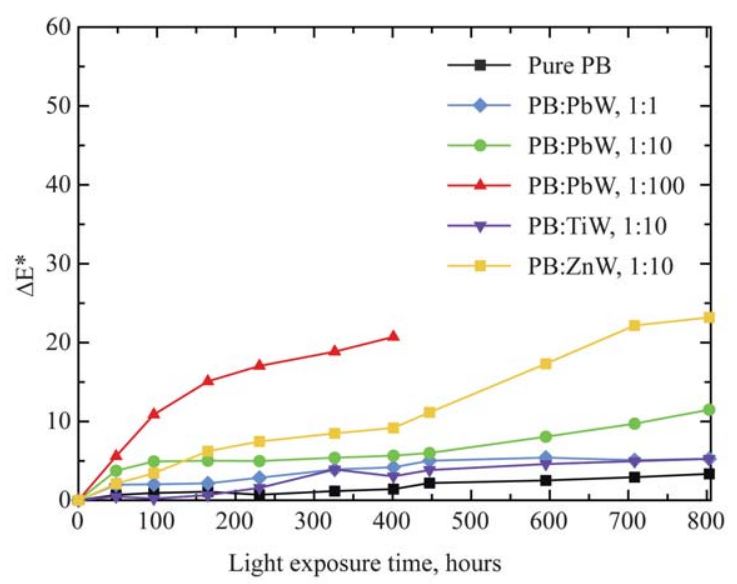

(b)

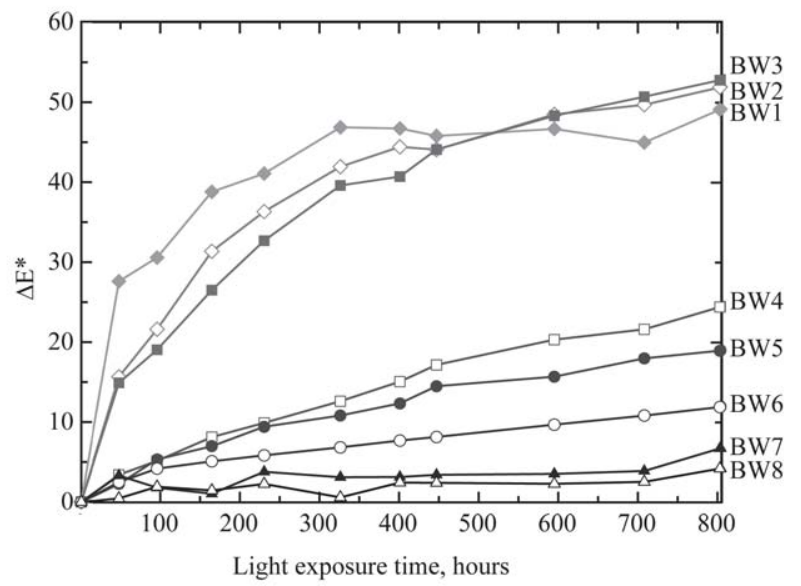

(d)

Fig. 1 Colour changes with light exposure time in the laboratory-synthesized, a, the commercial soluble, $\mathbf{b}$, the commercial insoluble, $\mathbf{c}$, Prussian blue, and the blue wool, BW1 to 8, standard samples, d. $\mathrm{PbW}$ is lead white, $\left(\mathrm{PbCO}_{3}\right)_{2} \mathrm{~Pb}(\mathrm{OH})_{2}$, TiW is titanium white, $\mathrm{TiO} \mathrm{O}_{2}$, and $\mathrm{ZnW}$ is zinc white, $\mathrm{ZnO}$.

in a $1: 1$ dilution ratio is as light fast as the blue wool standard 6 whereas the light fastness of the $1: 100$ dilution ratio is comparable with blue wool standards 3 or 4 . However, it should be noted that the Prussian blue concentration also decreased with higher dilution ratio. Among the samples with a $1: 10$ dilution ratio, all of which contained the same amount of Prussian blue for the three white pigments used, the $1: 10$ Prussian blue zinc oxide mixture exhibits the strongest sensitivity to irradiation, with a fair light fastness that is comparable with blue wool standard 4 . In contrast, the $1: 10$ dilution with titanium dioxide shows a very good light fastness that is comparable with blue wool standard 6 . The light fastness of the pure white pigments has also been investigated and is similar to the light fastness of blue wool standards 6 to 7 .

No significant regain of colour was observed in the samples placed in dark storage after $400 \mathrm{~h}$ of light exposure. Thus the colour loss appears irreversible in all the samples painted from linseed oil. Evidence of reversible fading has been reported in films exposed for relatively short periods before dark storage. . $^{19,31}$

The sensitivity of the samples to irradiation is more pronounced at the beginning of the exposure, as is clearly observed for the zinc white mixtures. This may explain why the discoloration increases abruptly when these samples are again subjected to the accelerated ageing after one week of dark storage.

The colour change for Prussian blue mixed with lead white at a $1: 100$ dilution ratio is smaller for the commercial soluble Prussian blue with $\Delta E^{*}=21(1)$ than for the laboratory-synthesized pigment with $\Delta E^{*}=32(1)$. This slightly improved light fastness of the commercial pigment and is presumably related to the industrial manufacturing process which may be better able to control the uniformity of the Prussian blue particle size. However, commercial and laboratory-synthesized Prussian blues generally exhibit similar light fastness behaviour. Moreover, the apparent solubility is not expected to play a role in the fading because both soluble and insoluble commercial Prussian blues exhibit a similar colour change evolution, see Fig. 1b and 1c. Cross-sections of the faded samples were prepared and observed under an Olympus BX51 optical microscope. The paint layer is about $20 \mu \mathrm{m}$ thick and appears to have a homogenous colour indicating a discoloration or fading of the entire paint layer.

UV-visible or optical spectroscopy has been performed on commercial soluble Prussian blue diluted with lead white and a loss of absorbance is clearly observed upon ageing, see Fig. 2. There also is a small shift in absorbance of approximately $50 \mathrm{~nm}$ 


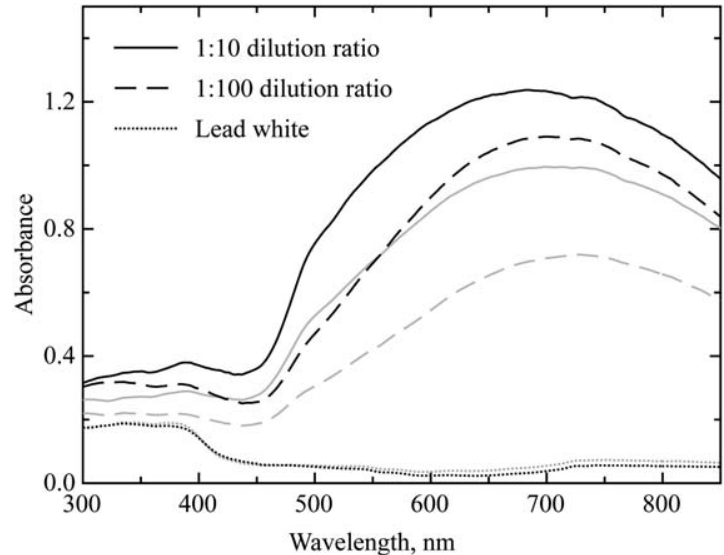

Fig. 2 UV-visible spectra of a commercial soluble Prussian blue, $\mathrm{KFe}^{\mathrm{II}}\left[\mathrm{Fe}^{\mathrm{II}}(\mathrm{CN})_{6}\right]$ mixed with lead white, $\left(\mathrm{PbCO}_{3}\right)_{2} \mathrm{~Pb}(\mathrm{OH})_{2}$, at $1: 10$ and $1: 100$ dilution ratios and of pure lead white, painted from linseed oil. The lighter curves correspond to the faded portion of the sample.

towards lower energy upon increasing the dilution ratio from $1: 10$ to $1: 100$ in both the unaged and aged samples. The UV-visible spectrum of pure lead white painted from linseed oil exhibits virtually no change upon sample ageing; as expected, above $400 \mathrm{~nm}$ the absorbance of lead white is very small.

\section{Iron ion electronic and structural configurations}

$\mathrm{X}$-ray absorption near edge spectroscopy. The iron K-edge X-ray absorption near edge spectra obtained on pure, non-aged and aged laboratory-synthesized Prussian blue, $\mathrm{KFe}^{\mathrm{II}}\left[\mathrm{Fe}^{\mathrm{II}}(\mathrm{CN})_{6}\right]$, mixed with lead white, $\left(\mathrm{PbCO}_{3}\right)_{2} \mathrm{~Pb}(\mathrm{OH})_{2}$, in $1: 10$ and $1: 100$ dilution ratios are shown in Fig. 3. The black curve represents the spectrum obtained in transmission mode on the pure soluble laboratory-synthesized Prussian blue. The remaining spectra have been obtained with fluorescence detection and differences in absorption intensity compared to the transmission spectrum are due to self-absorption effects. The weak intensity of the pre-edge peaks is indicative for the centrosymmetric $\mathrm{Fe}^{\mathrm{II}}$ and $\mathrm{Fe}^{\mathrm{III}}$ sites in soluble Prussian blue. ${ }^{32,28}$ The main K-edge and pre-edge positions have been determined from the maxima in the first derivative with an accuracy of about half the energy step, i.e., $0.26 \mathrm{eV}$ per datapoint. A shift in the absorption edge towards lower energy is observed upon ageing in the presence of the lead white, by approximately 0.2 and $2.5 \mathrm{eV}$ for the $1: 10$ and $1: 100$ dilution ratios, respectively. This shift corresponds either to a change in the density of states or to a decrease in the average iron oxidation state in the Prussian blue, i.e., an increase in the amount of low-spin $\mathrm{Fe}^{\mathrm{II}}$ as compared to high-spin $\mathrm{Fe}^{\mathrm{III}}$. The latter could suggest a partial reduction of Prussian blue, leading to a partial formation of Berlin white, i.e., iron(II) ferrocyanide, perhaps on the particle surfaces. However, the centroid of the pre-edge peaks do not show a significant shift towards lower X-ray energy, see the insets to Fig. 3, and does not support the hypothesis of a reduction.

The iron K-edge X-ray absorption near edge spectra were also obtained on unaged and aged insoluble commercial Prussian blue, $\mathrm{Fe}_{4}^{\mathrm{III}}\left[\mathrm{Fe}^{\mathrm{II}}(\mathrm{CN})_{6}\right]_{3}$, mixed with lead white, zinc white or titanium white in $1: 10$ dilution ratio. The spectra shown in Fig. 4 result from subtracting the unaged XANES spectrum from the aged spectrum. The importance of the difference in absorbance, $\Delta \mu$, can be directly correlated with the extent of the colour change, $\Delta E^{*}$, observed for these samples.

Extended X-ray absorption fine structure spectroscopy. The EXAFS spectra on the painted Prussian blue samples were recorded in fluorescence mode, which resulted in lower data quality compared to the transmission reference samples. As an example, the $k^{2}$ weighted EXAFS spectrum for the pure laboratory-synthesized soluble Prussian blue measured in transmission is compared in Fig. 6 with the fluorescence detection spectra for the unaged and aged pigment mixed with lead white in a $1: 100$ dilution ratio. The spectra for the pure powder and the unaged painted layer are identical. The EXAFS spectra of laboratory-synthesized and commercial non-aged Prussian blue painted layers mixed with lead white, zinc white and titanium

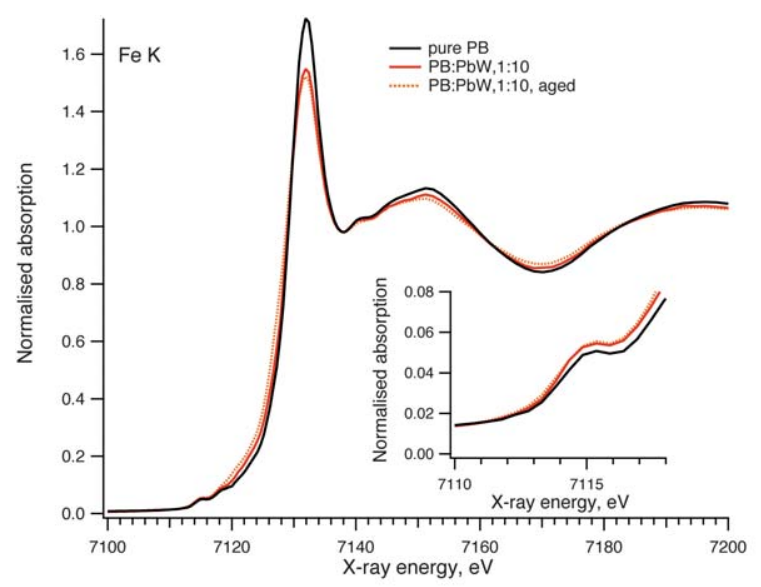

(a)

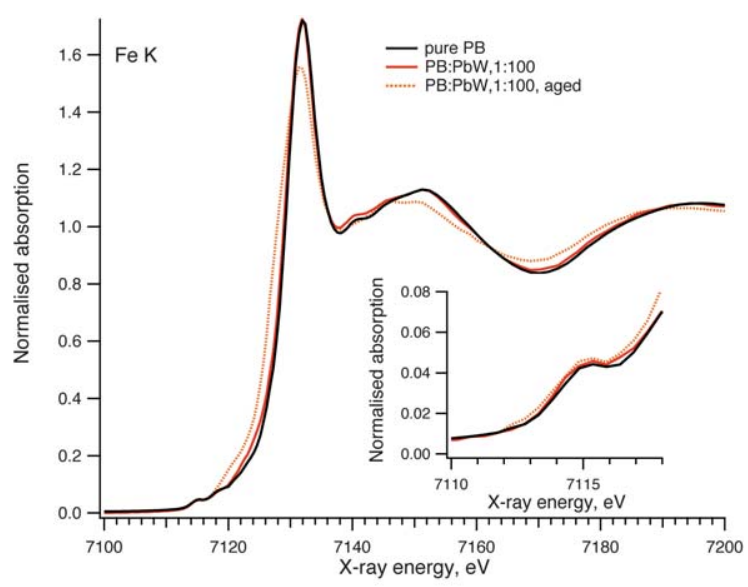

(b)

Fig. 3 Iron K-edge X-ray absorption near edge spectra of pure laboratory-synthesized Prussian blue, $\mathrm{KFe}^{\mathrm{III}}\left[\mathrm{Fe}^{\mathrm{II}}(\mathrm{CN})_{6}\right]$, powder painted from linseed oil with $\mathrm{PbW}$, lead white, $\left(\mathrm{PbCO}_{3}\right)_{2} \mathrm{~Pb}(\mathrm{OH})_{2}$, at a $1: 10$ dilution ratio, a, and at a $1: 100$ dilution ratio, $\mathbf{b}$. Inset: An expansion of the pre-edge peak spectral region. 


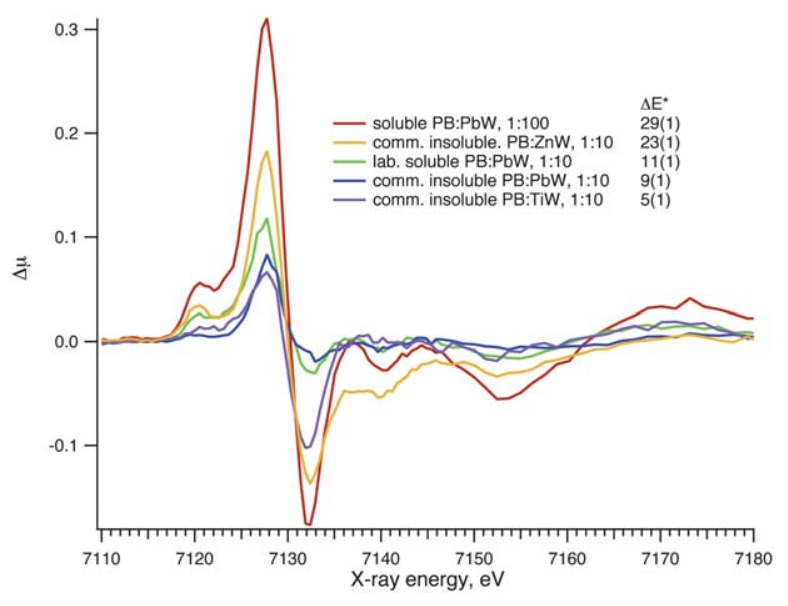

Fig. 4 Iron K-edge $\triangle$ XANES spectra of aged and unaged laboratorysynthesized Prussian blue, $\mathrm{KFe}^{\mathrm{II}}\left[\mathrm{Fe}^{\mathrm{II}}(\mathrm{CN})_{6}\right]$, painted from linseed oil with $\mathrm{PbW}$, lead white, $\left(\mathrm{PbCO}_{3}\right)_{2} \mathrm{~Pb}(\mathrm{OH})_{2}$, at a $1: 10$ and $1: 100$ dilution ratios and of insoluble commercial Prussian blue, $\mathrm{Fe}_{4}^{\mathrm{III}}\left[\mathrm{Fe}^{\mathrm{II}}(\mathrm{CN})_{6}\right]_{3}$, painted from oil with lead white, zinc white, $\mathrm{ZnO}$ and titanium white, $\mathrm{TiO}_{2}$ in $1: 10$ dilution ratio. The colour change $\Delta E^{*}$ for each sample is reported in the legend.

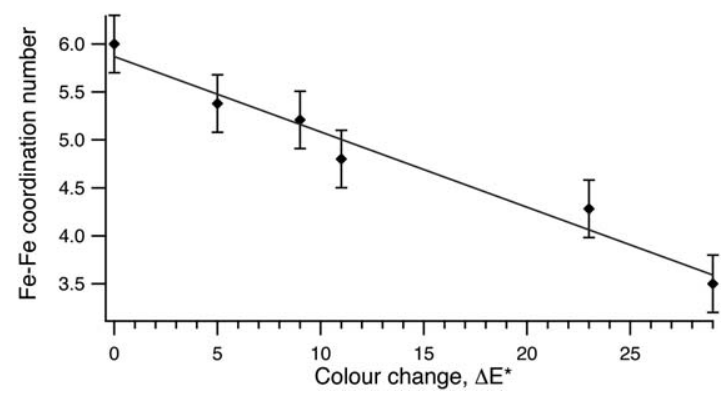

Fig. 5 The linear evolution of the Fe-Fe coordination number in aged painted Prussian blue pigments mixed with different white pigments as a function of colour change, $\Delta E^{*}$.

white in different dilution ratios were analysed and showed no significant differences. Bond distances and coordination numbers close to the crystal X-ray structure values were obtained by fixing the Debye-Waller factor to the average value obtained on the transmission EXAFS samples from Table 1. The average fit values for the five unaged painted samples are given in Table 2. The standard deviations are representative for the fit accuracy taking into account the data quality, the fit routine and systematic errors due to differences in background subtraction and data reduction. These standard deviations represent, therefore, our estimates for the errors in the EXAFS fits on the aged samples. For the EXAFS fit on the aged painted layers, the fit model on the corresponding non-aged paint layer was used as starting model and the bond distances, inner potential corrections and coordination numbers were optimised. The EXAFS fit on the aged laboratory synthesized soluble Prussian blue painted with lead white in a 1:100 dilution ratio is represented in Fig. 7. In general there was no change in bond distances, but a decrease in the $\mathrm{Fe}-\mathrm{Fe}$ coordination number with increasing colour change, $\Delta E^{*}$, was observed, see Fig. 5. A decrease in coordination number for the second $\mathrm{Fe}^{\mathrm{II}}-\mathrm{N}$ and $\mathrm{Fe}{ }^{\mathrm{III}}-\mathrm{C}$ shells was also observed for the aged laboratory-synthesized soluble Prussian blue paint layer mixed in lead white in a $1: 100$ ratio, see Table 2.

Iron-57 Mössbauer spectral results. The 85 and $295 \mathrm{~K}$ Mössbauer spectra of pure soluble laboratory-synthesized Prussian blue, $\mathrm{KFe}^{\mathrm{II}}\left[\mathrm{Fe}^{\mathrm{II}}(\mathrm{CN})_{6}\right]$, are shown in Fig. 8; the spectra obtained at 155 and $225 \mathrm{~K}$ are very similar. The Mössbauer spectral parameters resulting from a fit with two Lorentzian single lines are given in Table 4 . The spectra and the resulting fit parameters agree very well with those previously published for Prussian blue..$^{30,33}$ The absorption lines with negative and positive isomer shifts are assigned to low-spin iron(II) and high-spin iron(III), respectively. As the temperature decreases from 295 to $85 \mathrm{~K}$, both isomer shifts, as expected, become more positive as a result of the second-order Doppler shift. ${ }^{34}$ The relative areas of the iron(II) and iron(III) absorptions are temperature independent and, if the recoil-free fractions of iron(II) and iron(III) are assumed equal, a reasonable assumption at $85 \mathrm{~K},{ }^{35}$ the $A^{\mathrm{II}} / A^{\mathrm{III}}=48.5 / 51.5=0.94(4)$ ratio is in fair agreement with the ratio of one expected for a stoichiometric Prussian blue, $\left.\mathrm{KFe}^{\mathrm{II}}\left[\mathrm{Fe}^{\mathrm{II}} \mathrm{CN}\right)_{6}\right]$.
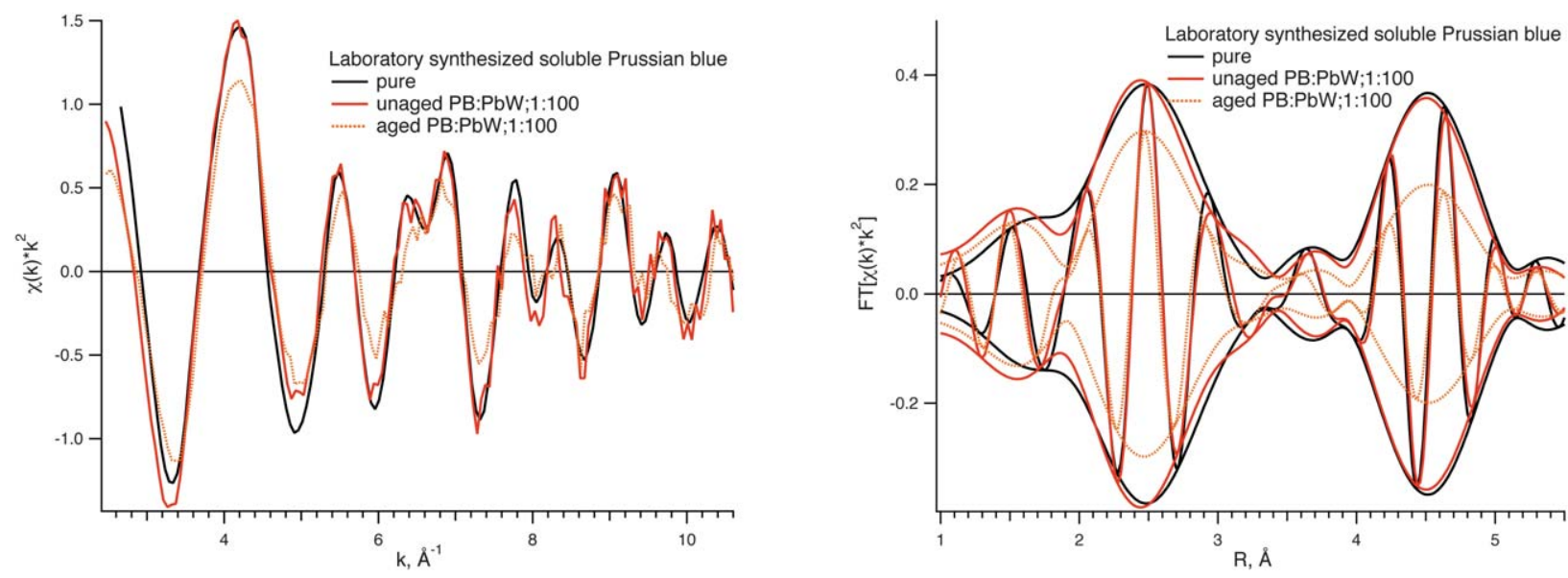

Fig. $6 \chi(k) * k^{2}$ and FT[ $\left.\chi(k) * k^{2}\right]$ spectra with $\Delta k \sim 5.3-10.5 \AA^{-1}$ for laboratory-synthesized soluble Prussian blue, in pure form and as unaged and aged painted layer mixed with lead white in a $1: 100$ dilution ratio. 
Table 2 Bond distances, $R$, coordination numbers, $N$, Debye-Waller factors, $\Delta \sigma^{2}$, and inner potential corrections, $\Delta E_{0}$, for the EXAFS fits on the five unaged Prussian blue paint layers and on the aged laboratory-synthesized Prussian blue pigment mixed with lead white in a $1: 100$ dilution ratio

\begin{tabular}{|c|c|c|c|c|c|c|c|c|c|c|}
\hline \multirow[b]{2}{*}{ Absorber-scatter } & \multicolumn{2}{|c|}{$\begin{array}{l}\text { Crystal } \\
\text { structure }\end{array}$} & \multicolumn{4}{|c|}{ EXAFS fit values for the unaged paint layers } & \multicolumn{4}{|c|}{ EXAFS fit values for the aged paint layer } \\
\hline & $R, \AA$ & $N$ & $R, \AA^{a}$ & $N^{a}$ & $\Delta \sigma^{2}, \AA^{2, a}$ & $\Delta E_{0}, \mathrm{eV}^{a}$ & $R, \AA$ & $N$ & $\Delta \sigma^{2}, \AA^{2}$ & $\Delta E_{0}, \mathrm{eV}$ \\
\hline $\mathrm{Fe}(\mathrm{II})-\mathrm{C}$ & $1.92(1)$ & 3 & $1.90(1)$ & $3.0(2)$ & 0.0002 & $1.0(3)$ & $1.91(1)$ & $3.2(2)$ & 0.0002 & $2(1)$ \\
\hline $\mathrm{Fe}(\mathrm{III})-\mathrm{N}$ & $2.04(1)$ & 3 & $2.11(1)$ & $3.0(1)$ & 0.0002 & $-0.5(8)$ & $2.13(1)$ & $3.2(1)$ & 0.0002 & $0.7(8)$ \\
\hline $\mathrm{Fe}(\mathrm{II})-\mathrm{N}$ & $3.04(1)$ & 3 & $3.05(1)$ & $3.0(1)$ & 0.0002 & $0.2(3)$ & $3.04(1)$ & $2.4(1)$ & 0.0002 & $-0.2(3)$ \\
\hline $\mathrm{Fe}(\mathrm{III})-\mathrm{C}$ & $3.16(1)$ & 3 & $3.15(1)$ & $2.9(1)$ & 0.0002 & $5.3(5)$ & $3.13(1)$ & $2.3(1)$ & 0.0002 & $4.5(5)$ \\
\hline
\end{tabular}

${ }^{a}$ Average values on the five unaged Prussian blue paint layers.
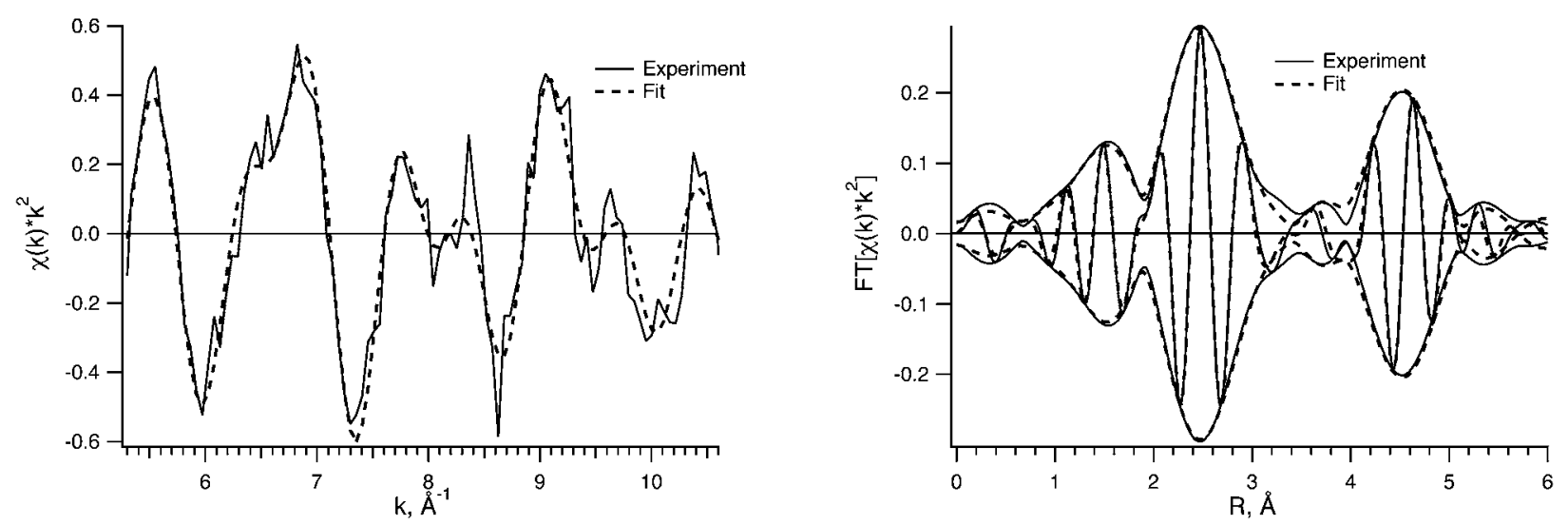

Fig. $7 \chi(k) * k^{2}$ and FT $\left[\chi(k) * k^{2}\right]$ spectra for the experimental data and fit for aged laboratory-synthesized soluble Prussian blue painted with lead white in a $1: 100$ dilution ratio with $\Delta k \sim 5.3-10.5 \AA^{-1}, \Delta R=1.0-5.2 \AA^{-1}, k^{2}$ variances: 1.51 (imaginary part) and 0.51 (absolute part).

Because the relative areas were found to be independent of temperature in the pure soluble Prussian blue, the Mössbauer spectra of the mixtures of Prussian blue with white lead, $(\mathrm{PbCO})_{2} \mathrm{~Pb}(\mathrm{OH})_{2}$, and zinc oxide, $\mathrm{ZnO}$, were only measured at $295 \mathrm{~K}$; the resulting spectra are shown in Fig. 8b and 8c, respectively. Because these Mössbauer spectra were obtained on material containing only a small amount of iron, the percent absorption is much smaller than in Fig. 8a. The Mössbauer spectral parameters resulting from a fit with two Lorentzian single lines are given in Table 3. It is immediately obvious that there is no change within the error limits in the isomer shifts of the iron(II) and iron(III) absorption lines, either upon mixing the pure Prussian blue with white lead or zinc oxide or upon ageing. Hence, the Mössbauer spectra reveal no reduction nor oxidation of the iron ions in the bulk of the Prussian blue pigments, a conclusion that seems to disagree with the hypotheses for

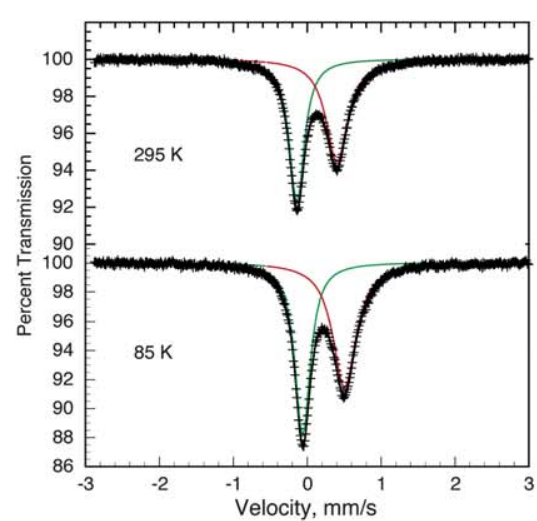

(a)

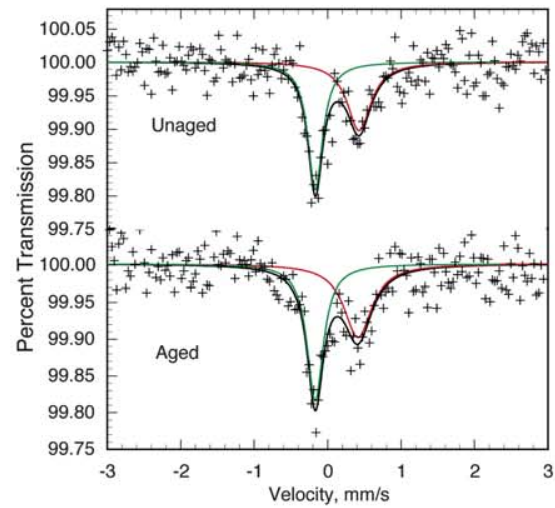

(b)

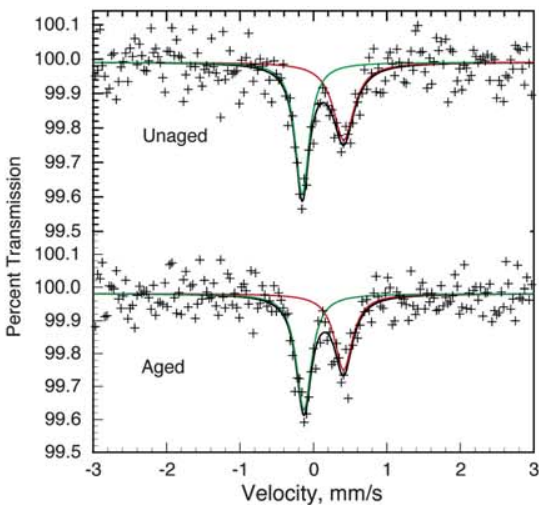

(c)

Fig. 8 The Mössbauer spectra of laboratory-synthesized $\mathrm{KFe}^{\mathrm{III}}\left[\mathrm{Fe}^{\mathrm{II}}(\mathrm{CN})_{6}\right]$ Prussian blue obtained at 85 and $295 \mathrm{~K}$, a; a $1: 100$ mixture of Prussian blue and white lead, $\left(\mathrm{PbCO}_{3}\right)_{2} \cdot \mathrm{Pb}(\mathrm{OH})_{2}$, unaged and aged, $\mathbf{b}$; a $1: 10$ mixture of $\mathrm{K}^{\mathrm{I}} \mathrm{Fe}^{\mathrm{III}}\left[\mathrm{Fe}^{\mathrm{II}}(\mathrm{CN})_{6}\right]$ Prussian blue and zinc oxide, $\mathrm{ZnO}$, unaged and aged, c. The green and red solid lines represent the iron(II) and iron(III) spectral components, respectively. 
Table 3 Mössbauer spectral parameters of Prussian blue and its mixtures

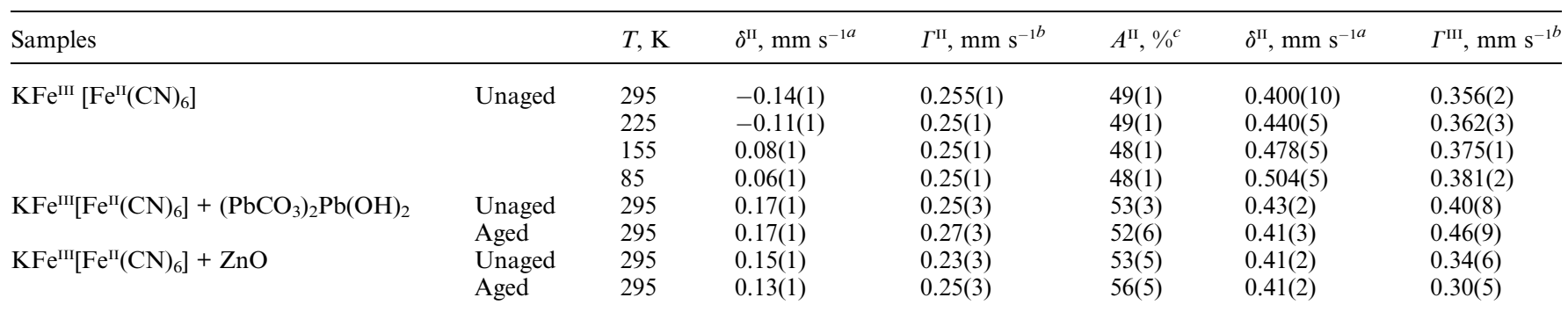

${ }^{a}$ The isomer shift, $\delta$, is referred to $\alpha$-iron at $295 \mathrm{~K} .{ }^{b} \Gamma$ is the full linewidth at half-height. ${ }^{c} A^{\mathrm{III}}=100-A^{\mathrm{II}}$.

fading proposed in the literature. ${ }^{1}$ The only barely significant change in the Mössbauer spectra parameters is the $A^{\mathrm{II}} / A^{\mathrm{III}}$ ratio that increases to $c a .53 / 47=1.1(2)$ upon mixing with the white pigments and remains unchanged upon ageing.

Attenuated total reflectance infrared spectra. The attenuated total reflectance Fourier transform infrared spectra, obtained between 2200 and $1000 \mathrm{~cm}^{-1}$, on laboratory-synthesized soluble Prussian blue, $\mathrm{KFe}^{\mathrm{II}}\left[\mathrm{Fe}^{\mathrm{II}}(\mathrm{CN})_{6}\right]$, mixed with lead white, $\mathrm{Pb}$ $\left(\mathrm{CO}_{3}\right)_{2} \mathrm{~Pb}(\mathrm{OH})_{2}$, at $1: 10$ and $1: 100$ dilution ratios, are shown in Fig. 9. The lighter curve corresponds to the aged portion of the sample. Soluble Prussian blue is characterized by a single peak at $2088 \mathrm{~cm}^{-1}$ that corresponds to the $\mathrm{CN}$ stretching vibration. ${ }^{36}$ The other peaks in the infrared spectrum correspond either to the binder or to the white pigment that has been combined with the Prussian blue.

The band at $1735 \mathrm{~cm}^{-1}$ is assigned to the ester carbonyl vibrational absorption arising from the linseed oil used as binder. The spectral features at $1238 \mathrm{w}, 1162 \mathrm{~m}$ and $1095 \mathrm{~m} \mathrm{~cm}^{-1}$ are assigned to $\mathrm{CO}$ stretching modes and are also characteristic of the triglyceride ester linkages found in linseed oil. The narrow peak at $1042 \mathrm{w} \mathrm{cm}^{-1}$ is assigned to the symmetric $\mathrm{CO}_{3}$-stretching vibration of lead white, whereas the broad intense peak at 1390 $\mathrm{cm}^{-1}$ is assigned to the antisymmetric $\mathrm{CO}_{3}$-stretching vibration. Lead white is known to accelerate the drying and ageing of linseed oil and to produce a hard and resistant film with more durable and stable properties. This film is commonly believed to result from the formation of metal carboxylates, induced by the saponification of esters and the deprotonation of carboxylic acids. The presence of lead white thus influences the ageing of linseed oil by causing the hydrolysis of its triglycerides. This is indicated by a loss of absorbance for the ester linkages at 1735 , 1238, 1162, and $1095 \mathrm{~cm}^{-1}$ in the spectra obtained after ageing. ${ }^{37,38}$ The areas of the carbonate absorptions at $1042 \mathrm{w}$ and $1390 \mathrm{~s}, \mathrm{br} \mathrm{cm}^{-1}$ decrease slightly with ageing. This decrease can be attributed to the displacement of the carbonate ligands from the lead ions by the carboxylate anions derived from the esters and acids. However the rate of decrease of the carbonate absorption depends on the concentration of lead white and is almost negligible when the proportion of lead white is important in comparison with the amount of linseed oil. In this study, the paint layers have been prepared with consideration of the oil absorption indexes of Prussian blue and lead white, i.e., approximately 100 and 9 to $13 \mathrm{wt} \%$, respectively. The quantity of oil in mixtures of the Prussian blue and lead white at $1: 10$ and $1: 100$ dilution ratios is thus about 20 and $13 \mathrm{wt} \%$ of the total weight of pigments, respectively. Because of the small amount of oil in the samples, the intensity of the carbonate absorption remains unaffected through ageing. This band can be considered as an indirect calibration that allows a comparison between the

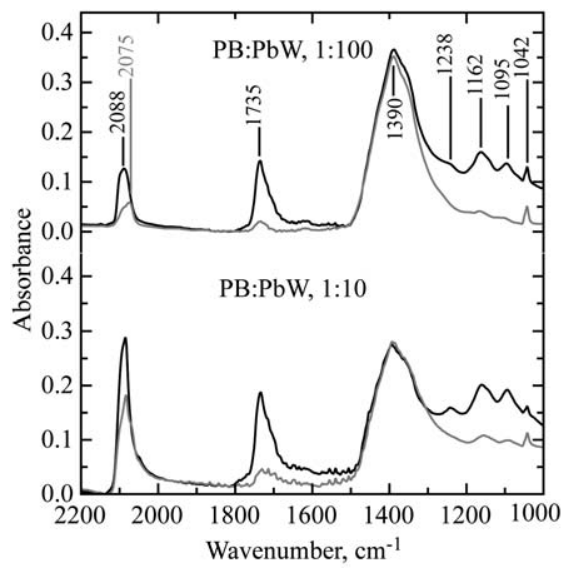

(a)

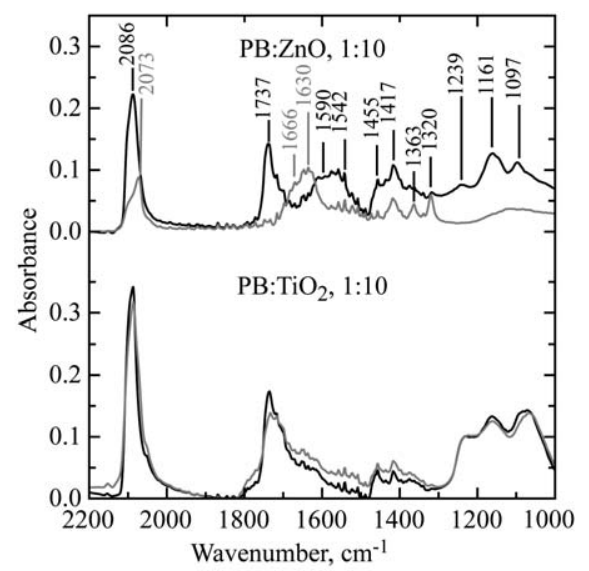

(b)

Fig. 9 The attenuated total reflectance infrared spectra of laboratory-synthesized $\mathrm{KFe}^{\mathrm{III}}\left[\mathrm{Fe}^{\mathrm{II}}(\mathrm{CN})_{6}\right]$ Prussian blue mixed with $\mathrm{PbW}$, lead white, $\left(\mathrm{PbCO}_{3}\right)_{2} \mathrm{~Pb}(\mathrm{OH})_{2}$, at $1: 10$ and $1: 100$ dilution ratios, a; and commercial Prussian blue mixed with zinc white, $\mathrm{ZnO}$, and titanium white, TiO ${ }_{2}$, in a $1: 10$ dilution ratio, $\mathbf{b}$. The gray curve corresponds to the aged part of the sample. 
aged and unaged spectra. Any other loss of absorbance is thus of quantitative significance.

It is noteworthy that the intensity of the $\mathrm{CN}$ vibrational band at $2088 \mathrm{~cm}^{-1}$ dramatically decreases upon ageing, whatever the dilution. Because the molar concentration of the Prussian blue has not varied during ageing, this loss of absorbance indicates an electronic change in the Prussian blue, a change which leads to a slightly different intervalent charge transfer pathway and, as a consequence, a reduced extinction coefficient. Aside from the loss of absorbance, it is worth noting that the shape of the $\mathrm{CN}$ stretching band changes after ageing, see Fig. 9. For dilution of Prussian blue with lead white in a $1: 10$ ratio, a shoulder appears at lower energy at $2072 \mathrm{~cm}^{-1}$. For dilution at a $1: 100$ ratio, two contributions at 2088 and $2075 \mathrm{~cm}^{-1}$ are observed in the CN stretching band, with the maximum in the absorbance shifted towards lower energy. The frequency of the $\nu(\mathrm{CN})$ band depends on the strength of the bond with the central cation. In aqueous solution, the stretching mode for free $\mathrm{CN}^{-}$occurs at $2060 \mathrm{~cm}^{-1}$, whereas the $\nu(\mathrm{CN})$ frequency increases upon coordination to a metal ion. The position of this mode depends on the electronegativity, the oxidation state and the coordination number of the metal. ${ }^{36}$ Lower frequency bands can be assigned to a $\nu(\mathrm{CN})$ mode associated with iron(II) whereas the $\nu(\mathrm{CN})$ mode associated with iron(III) is characterized by a higher frequency at 2155 $\mathrm{cm}^{-1} .39,40$ The shift towards lower energies observed in the infrared spectra after ageing indicates the formation of increased amounts of iron(II) ferrocyanide, a compound that is characterized by a band of lower intensity in comparison to Prussian blue. ${ }^{13}$

The infrared spectra of commercial insoluble Prussian blue $\mathrm{Fe}_{4}^{\mathrm{III}}\left[\mathrm{Fe}^{\mathrm{II}}(\mathrm{CN})_{6}\right]_{3}$ mixed with zinc white, $\mathrm{ZnO}$, and titanium white, $\mathrm{TiO}_{2}$, between 2200 and $1000 \mathrm{~cm}^{-1}$ are shown in Fig. 9. The absorption bands that characterize titanium dioxide and zinc oxide occur below $700 \mathrm{~cm}^{-1}$ and therefore have not been observed in this study. The spectra of Prussian blue mixed with zinc white dramatically change upon ageing. Similar features, such as the loss of absorbance and shift towards lower energy have been observed for the $\nu(\mathrm{CN})$ mode. Upon ageing the intensity of the carbonyl band at $1737 \mathrm{~cm}^{-1}$ decreases substantially as is also the case for the broad carboxylate absorptions at 1590 and $1542 \mathrm{~cm}^{-1}$ and the $\mathrm{CH}$ bending vibrations at 1455, 1417 and $1363 \mathrm{~cm}^{-1}$. Moreover a new band at $1630 \mathrm{~cm}^{-1}$ appears, a band which can be assigned to the antisymmetric COOstretching mode and may be attributed to the formation of oxalates. The band at $1666 \mathrm{~cm}^{-1}$ indicates the formation of zinc carboxylates. ${ }^{41}$ These new features are a consequence of the tendency of zinc oxide to form a soap with the free acids found in oils. ${ }^{42}$ In contrast, in the titanium white mixture there is no significant modification in the infrared spectrum upon ageing. This is consistent with the very good light fastness observed for this mixture, see Fig. 1. Rutile titanium dioxide is chemically inert and does not react with its linseed oil medium or with other pigments. ${ }^{18}$

\section{Discussion}

Because pure Prussian blue painted from linseed oil has an excellent light fastness, the fading of Prussian blue in a linseed oil medium must be related to the presence of an additional pigment.
The admixture of a white pigment strongly affects the permanence of Prussian blue under light exposure, although the strength of the discoloration depends on the nature of the white pigment.

Based upon the iron K-edge X-ray absorption, Mössbauer and infrared spectral results, one can exclude iron oxidation as being responsible for the fading of Prussian blue in its mixtures with white pigments. The unchanged $\mathrm{Fe}{ }^{\mathrm{II}} / \mathrm{Fe}$ III bulk ratio upon ageing, the loss of absorbance of the $\nu(\mathrm{CN})$ band and the change in the nature of the $\mathrm{Fe}^{\mathrm{II}}-\mathrm{C}-\mathrm{N}-\mathrm{Fe}^{\mathrm{III}}$ electron transfer pathway observed in the X-ray absorption spectra suggest a rupture of a fraction of $\mathrm{Fe}^{\mathrm{II}}-\mathrm{C}-\mathrm{N}-\mathrm{Fe}^{\mathrm{III}}$ sequences, leading to a weakening of the intervalent charge transfer between the $\mathrm{Fe}^{\mathrm{II}}$ and $\mathrm{Fe}^{\mathrm{III}}$ ions. The underlying mechanism of the possible change in the intervalent transfer pathway has not yet been identified. The possibility of ion exchange between the weakly bond $\mathrm{N}$-coordinated iron(III) ions and other metal ions, as suggested in electrochemical studies, ${ }^{17}$ does not seem to be a realistic hypothesis for understanding the colour loss in Prussian blue and white pigments mixtures. The unlikely substitution reaction between the Prussian blue iron(III) ions and the lead(II) and zinc(II) ions of the white pigments would have lead to a far larger variation in both the X-ray absorption spectra and their corresponding Fourier transforms than has been observed. The decrease in the coordination number could, however, be indicative of the creation of defects in the Prussian blue crystals. The loss of coordinated water may in part lead to these defects and the observed decrease in the iron coordination number.

At this point of the discussion, the hypothesis of a partial reduction may not be excluded in view of the XANES and infrared results. Because the Mössbauer spectral parameters remain unchanged upon ageing, this reduction must necessarily occur at the surface of the paint layer. Although optical photomicrographs reveal a homogenous coloured layer after ageing, it is reasonable to consider that the chemical change upon light illumination takes place first in the Prussian blue at the surface before appearing in the deeper layers. Fading at the top of a thick paint layer containing Prussian blue and lead white has been previously observed, ${ }^{19}$ one can thus assume that surface discoloration in Prussian blue paint layers occurs prior to any bulk colour change. This phenomenon could explain why the attenuated total reflectance infrared spectra, with a probe depth of only $1 \mu \mathrm{m}$, shows more noticeable differences upon ageing than do the other techniques used herein.

If Prussian blue is effectively partially reduced, it most likely occurs because of the presence of the white pigment and the light irradiation. Both photoinduced reduction or oxidation in Prussian blue are made possible when Prussian blue is coupled with a material that generates electrons upon UV or visible light irradiation. ${ }^{15}$ This particular feature has been exploited to produce photoswitchable magnetic materials. Prussian blue also exhibits ferromagnetic behavior below its Curie temperature whereas the reduced or oxidized forms of Prussian blue are paramagnetic even at the low temperatures at which Prussian blue is magnetically ordered. ${ }^{43}$ Reduction in a Prussian blue paint layer may thus occur in the presence of a species that can provide electrons upon irradiation. The provenance of these electrons remains unclear. Because of their basic character, lead white and zinc oxide tend to form a soap by interacting with the linseed oil 
fatty acids and thus, enhance the oxidative polymerisation of the oil upon ageing. ${ }^{44}$ The presence of these basic pigments in linseed oil may favour the production of electrons that will initiate the reduction of Prussian blue. In contrast, titanium white, $\mathrm{TiO}_{2}$, is amphoteric, with a predominance of acid properties. This pigment does not influence the ageing of oil, as is observed in the infrared spectrum shown at the bottom of Fig. 9, and the absence of this influence may explain the good light fastness observed for Prussian blue mixed with titanium white. The predominance of one mechanism on the other, i.e., decrease in the iron coordination number versus partial reduction of Prussian blue, still needs to be clarified.

Aside light absorption, diffusion of the radiation in the samples should also be considered. Diffusion in a paint layer is enhanced by smaller particle size of pigments. Titanium white is made of smaller particles, varying from 0.2 to $0.5 \mu \mathrm{m}$ in size, than zinc or lead whites, whose pigment particles are approximately from 0.25 to $1 \mu \mathrm{m}$ and from 1 to $2 \mu \mathrm{m}$, respectively. Diffusion of light is thus increased in the paint layers containing Prussian blue and titanium white. However this does not seem to play an important role in the discoloration processes because samples prepared with titanium white exhibit no colour change.

\section{Conclusions}

The light fastness of modern Prussian blue pigments is dramatically decreased in mixtures with certain white pigments. The investigation of the local electronic and structural configurations of the iron ions reveals a disruption in the intervalent electron transfer $\mathrm{Fe}^{\mathrm{II}}-\mathrm{C}-\mathrm{Fe}^{\mathrm{III}}$ pathway upon ageing. A decrease in the iron coordination number in the second and third shells has been evidenced in aged Prussian blues, leading to a weakening of the intervalent charge transfer between the $\mathrm{Fe}^{\mathrm{II}}$ and $\mathrm{Fe}^{\mathrm{III}}$ ions. According to the results obtained by X-ray absorption and infrared spectroscopy, a partial reduction of the surface iron ions in Prussian blue into iron(II) ions to form surface iron(II) ferrocyanide may be a complementary explanation for the fading. However the proportion of iron(III) ions reduced to iron(II) in the bulk cannot exceed a few percent because no change in the $\mathrm{Fe}$ (II)/Fe(III) ratio was observed by Mössbauer spectroscopy upon ageing. The nature of the white pigment is a determining factor in the degree of fading of Prussian blue mixtures with white pigments. The increased discoloration of Prussian blue mixed with lead white or zinc white may be related to the accelerated oxidative polymerisation of linseed oil in the presence of pigments that tend to form soaps with the free acids in oil.

This hypothesis must be confirmed by further investigating the influence of the binder as well as the acidic or basic character of the added pigment on the light fastness of Prussian blue. Analyses by high resolution X-ray diffraction would be considered in order to evaluate the evolution of long-range packing, grain size and microstructure in the paint layers through ageing. Electron paramagnetic resonance measurements could also help to understand the discoloration mechanisms through the detection of free radicals induced by light radiation in the paint layers. Finally, we have also applied Raman spectroscopy to perform extensive measurements on unaged and aged mixtures. The conclusions are similar and confirm those proposed in the present paper but some points still need to be clarified. The final results obtained from Raman spectroscopy will be presented in a forthcoming publication.

\section{Acknowledgements}

The financial support of the Fonds National de la Recherche Scientifique (F.R.S-FNRS), Belgium (grants 9.456595 and 1.5.064.05), is gratefully acknowledged. This work is supported by the Belgian inter-university Attraction Pole Program P6/16 Belgian State Belgian Science Policy. G. Silversmit is supported by a postdoctoral fellowship from the Research FoundationFlanders (FWO-Vlaanderen, Belgium).

\section{References}

1 J. Kirby and D. Saunders, Nat. Gall. Tech. Bull., 2004, 25, 73-99.

2 H. W. Levison, Artists' Pigments Lightfastness Tests and Ratings, Colorlab, Hallandale, Florida, 1976.

3 S. Ferlay, Nature, 1995, 378, 701-703.

4 M. Verdaguer, A. Bleuzen, V. Marvaud, J. Vaissermann, M. Seuleiman, C. Desplanches, A. Scuiller, C. Train, R. Garde, G. Gelly, C. Lomenech, I. Rosenman, P. Veillet, C. Cartier and F. Villain, Coord. Chem. Rev., 1999, 190-192, 1023-1047.

5 V. D. Neff, J. Electrochem. Soc., 1978, 125, 886-887.

6 R. J. Emrich, L. Traynor, W. Gambogi and E. Buhks, J. Vac. Sci. Technol., A, 1987, 5, 1307-1310.

7 N. M. Rowley and R. J. Mortimer, Sci. Prog., 2002, 85, 243-262.

8 Q. Chi and S. Dong, Anal. Chim. Acta, 1995, 310, 429-436.

9 R. Koncki and O. S. Wolfbeis, Biosens. Bioelectron., 1999, 14, 87-92.

10 M. B. Robin, Inorg. Chem., 1962, 1, 337-342.

11 R. J. Mortimer, Chem. Soc. Rev., 1997, 26, 147-156.

12 J. F. Duncan and P. W. R. Wigley, J. Chem. Soc., 1963, 1120-1125.

13 P. J. Kulesza, M. A. Malik, A. Denca and J. Strojek, Anal. Chem., 1996, 68, 2442-2446.

14 A. Xidis and V. D. Neff, J. Electrochem. Soc., 1991, 138, 3637-3642.

15 M. Taguchi, I. Yagi, M. Nakagawa, T. Iyoda and Y. Einaga, J. Am. Chem. Soc., 2006, 128, 10978-10982.

16 Y. Einaga and Y. Einaga, Chem. Mater., 2002, 14, 4846-4850.

17 A. Dostal, M. Hermes and F. Scholz, J. Electroanal. Chem., 1996, 415, 133-141.

18 M. Laver, Artist's Pigments, A Handbook of Their History and Characteristics, Oxford Universtiy Press, New York, 1997, vol. 3, pp. 295-355.

19 J. Kirby, Nat. Gall. Tech. Bull., 1993, 14, 62-71.

20 G. Wyszecki and W. Stiles, Color Science, Concepts and Methods, Quantitative Data and Formulae, John Wiley and Sons, INC., New York, 2nd edn, 2000.

21 S. Nikitenko, A. M. Beale, A. M. J. van der Eerden, S. D. M. Jacques, O. Leynaud, M. O'Brien, D. Detollenaere, R. Kaptein, B. M. Weckhuysen and W. Bras, J. Synchrotron Radiat., 2008, 15, $632-640$.

22 M. Vaarkamp, J. Linders and D. Koningsberger, Phys. B, 1995, 208209, 159-160.

23 M. Vaarkamp, I. Dring, R. Oldman, E. Stern and D. Koningsberger, Phys. Rev. B: Condens. Matter, 1994, 50, 7872-7883.

24 J. Cook and D. Sayers, J. Appl. Phys., 1981, 52, 5024-5031.

25 D. Koningsberger, B. Mojet, G. van Dorsen and D. Ramaker, Top. Catal., 2000, 10, 143-155.

26 A. Ankudinov, B. Ravel, J. Rehr and S. Conradson, Phys. Rev. B: Condens. Matter Mater. Phys., 1998, 58, 7565-7576.

27 P. Glatzel, L. Jacquamet, U. Bergmann, F. de Groot and S. Cramer, Inorg. Chem., 2002, 41, 3121-3127.

28 H. Buser, D. Schwarzenbach, W. Petter and A. Ludi, Inorg. Chem., 1977, 16, 2704-2710.

29 M. J. Willans, R. E. Wasylishen and R. McDonald, Inorg. Chem., 2009, 48, 4342-4353.

30 E. Reguera, J. Fernández-Bertrán, A. Dago and C. Díaz, Hyperfine Interact., 1992, 73, 295-308.

31 M. Ware, Cyanotype: The History, Science and Art of Photographic Printing in Prussian blue, Science Museum, London, 1999, p. 120. 
32 M. Wilke, F. Farges, P.-E. Petit, G. E. BrownJr and F. Martin, Am. Mineral., 2001, 86, 714-730.

33 K. Maer Jr., M. L. Beasley, R. L. Collins and W. O. Milligan, J. Am. Chem. Soc., 1968, 90, 3201-3208.

34 G. K. Shenoy, F. E. Wagner and G. M. Kalvius, Mössbauer isomer shift, 1978, 49.

35 A. Ito, M. Suenaga and K. Ono, J. Chem. Phys., 1968, 48, 3597-3599.

36 K. Nakamoto, Infrared and Raman Spectra of Inorganic and Coordination Compounds, Wiley-Interscience Publication, New York, 1978, p. 259.

37 J. Van der Weerd, A. van Loon and J. J. Boon, Stud. Conserv., 2005, 50, $1-20$.

38 R. J. Meilunas, J. G. Bentsen and A. Steinberg, Stud. Conserv., 1990, 35, 33-51.
39 C. W. Ng, J. Ding, Y. Shi and L. M. Gan, J. Phys. Chem. Solids, 2001, 62, 767-775.

40 S. A. Agnihotry, P. Singh, A. G. Joshi, D. P. Singh, K. N. Sood and S. M. Shivaprasad, Electrochim. Acta, 2006, 51, 42914301.

41 R. Arbizzani, U. Casellato, E. Fiorin, L. Nodari, U. Russo and P. A. Vigato, J. Cult. Heritage, 2004, 5, 167-182.

42 H. Kühn, Artists' Pigments, A Handbook of Their History and Characteristics, Oxford University Press, New York, 1985, vol. 1, p. 175.

43 T. Yamamoto, N. Saso, Y. Umemura and Y. Einaga, J. Am. Chem. Soc., 2009, 131, 13196-13197.

44 M. Cotte, E. Checroun, J. Susini and P. Walter, Appl. Phys. A: Mater. Sci. Process., 2007, 89, 841-848. 